\title{
COMMENTARY
}

\section{Urine sTREM-1 assessment in diagnosing sepsis and sepsis-related acute kidney injury}

\author{
Marc Derive and Sébastien Gibot* \\ See related research by Su et al., http://ccforum.com/content/15/5/R250
}

\begin{abstract}
The triggering receptor expressed on myeloid cells-1

(TREM-1) is an immunoreceptor whose role is to amplify the inflammatory response mediated by the engagement of Toll-like and NOD-like receptors. As the expression of TREM-1 is believed to be upregulated during infection, this protein has been studied as a sepsis biomarker. In the previous issue of Critical Care, Su and colleagues reported on the usefulness of urinary soluble TREM-1 in diagnosing sepsis and assessingits severity. Importantly, the authors describe, for the first time, that urinary soluble TREM1 measurement is able to predict the development of sepsis-associated acute kidney injury (AKI). If these results were to be confirmed by larger studies, urinary soluble TREM-1 would possibly become a new biomarker for sepsis-associated AKI.
\end{abstract}

In the previous issue of Critical Care, Su and colleagues [1] reported on the usefulness of measuring urinary concentrations of the soluble form of the triggering receptor expressed on myeloid cells-1 (sTREM-1) during sepsis. One hundred four patients were enrolled in the authors' study: 16 presented with systemic inflammatory response syndrome, 35 with sepsis, and 53 with severe sepsis/septic shock.

The authors demonstrated that the urinary sTREM-1 concentration was associated with the positive diagnosis of sepsis with an acceptable area under the receiver operating characteristic curve $(0.797,95 \%$ confidence interval 0.711 to 0.884 ). TREM- 1 is a member of the immunoglobulin superfamily expressed mainly on mature monocytes, macrophages, and neutrophils. Its engagement leads to an amplification of the inflammatory response mediated by the activation of Toll-like and

${ }^{*}$ Correspondence: s.gibot@chu-nancy.fr

Service de Réanimation Médicale, Hopital Central, 54035 Nancy Cedex, France

Biomed Central o 2011 Biomed Central Ltd
NOD-like receptors [1,2]. A soluble form of TREM-1 appears after its cleavage from the cell's surface through the action of matrix metalloprotease-9 [3]. sTREM-1 has been studied as a sepsis biomarker, but investigations of its plasma concentration have had contrasting results. Indeed, a recent meta-analysis showed an $82 \%$ sensitivity and an $86 \%$ specificity in this setting [4]. All in all, plasma sTREM-1 proved to be equivalent to procalcitonin in diagnosing sepsis. More interesting is the fact that the evaluation of local production of STREM-1, at the site of presumed infection, is associated with better performances: this has been studied during pneumonia, arthritis, pancreatitis, peritonitis, and meningitis [5-9].

In the study by $\mathrm{Su}$ and colleagues, the urinary sTREM-1 concentration was able to predict sepsis with a good specificity (93\%) but a modest sensitivity (64\%). This low sensitivity may be explained by the fact that urinary sTREM-1 measurement has been found to be inaccurate for the diagnosis of urinary sepsis [10]: about $30 \%$ of the patients reported by $\mathrm{Su}$ and colleagues presented with this diagnosis.

The authors went on to investigate the prognosis value of urinary sTREM-1 concentration and found that, in comparison with survivors, non-survivors exhibited a permanent increase of sTREM-1. Again, the plasma level of sTREM-1 has been repeatedly associated with prognosis or severity: the current study shows that its urinary measurement may also be interesting in this context.

The major finding of $\mathrm{Su}$ and colleagues is that urinary sTREM-1 measurement is able to predict the development of sepsis-associated acute kidney injury (AKI). Indeed, when sTREM-1 was measured within 48 hours before the diagnosis of AKI, its elevation was associated with a $94 \%$ sensitivity and a $76 \%$ specificity in predicting the occurrence of this condition. This is the first report showing that sTREM-1 may predict AKI.

This poses several questions. First, where does urinary STREM-1 originate from? Measured in the urine, sTREM-1 may reflect a systemic production and may correlate with its plasma concentration: as sTREM- 1 is a $27-\mathrm{kDa}$ protein, it can be excreted by the kidney provided that kidney injury exists. But sTREM-1 could also be 
produced locally by the endothelial cells or the infiltrating inflammatory cells that are recruited during acute tubular necrosis or both. Unfortunately, the plasma concentrations of sTREM- 1 are not described in the current study and therefore it is impossible to draw any conclusions regarding the origin of urinary sTREM-1 during AKI.

Several other biomarkers have been investigated for the diagnosis of sepsis-associated AKI. The measurement of plasma neutrophil gelatinase-associated lipocalin (NGAL), for example, was able to predict AKI progression during sepsis with an acceptable performance [11]. Therefore, it would be interesting to compare the sTREM-1 performance with that of these other markers. Finally, as underlined by the authors, only 17 out of 88 patients with sepsis developed an AKI after 48 hours of admission. This very low sample size warrants caution in interpreting the results of the study, and confirmatory work is needed.

In their study, Su and colleagues confirm the usefulness of measuring sTREM-1 concentration in diagnosing sepsis and assessing its severity. They also extend the current knowledge on TREM-1 in showing that its soluble form, when measured in the urine, was able to predict the development of AKI. If these results were to be confirmed by larger studies, urinary sTREM-1 would possibly become a new biomarker for sepsis-associated AKI.

\section{Abbreviations}

AKI, acute kidney injury; sTREM-1, soluble form of the triggering receptor expressed on myeloid cells-1; TREM-1, triggering receptor expressed on myeloid cells-1.

\section{Competing interests}

The authors declare that they have no competing interests.

Published: 30 November 2011

\section{References}

1. Su L, Feng L, Zhang J, Xiao Y, Jia Y, Yan P, Feng D, Xie L: Diagnostic value of urine sTREM-1 for sepsis and relevant acute kidney injuries: a prospective study. Crit Care 2011, 15:R250.
2. Gibot $\mathrm{S}$ : Clinical review: role of triggering receptor expressed on myeloid cells-1 during sepsis. Crit Care 2005, 9:485-489.

3. Gómez-Piña V, Soares-Schanoski A, Rodríguez-Rojas A, Del Fresno C, García F, Vallejo-Cremades MT, Fernández-Ruiz I, Arnalich F, Fuentes-Prior P, LópezCollazo E: Metalloproteinases shed TREM-1 ectodomain from lipopolysaccharide-stimulated human monocytes. J Immunol 2007, 179:4065-4073.

4. Jiyong J, Tiancha H, Wei C, Huahao S: Diagnostic value of the soluble triggering receptor expressed on myeloid cells-1 in bacterial infection: a meta-analysis. Intensive Care Med 2009, 35:587-595.

5. Gibot S, Cravoisy A, Levy B, Bene MC, Faure G, Bollaert PE: Soluble triggering receptor expressed on myeloid cells and the diagnosis of pneumonia. NEngl J Med 2004, 29:451-458.

6. Collins CE, La DT, Yang HT, Massin F, Gibot S, Faure G, Stohl W: Elevated synovial expression of triggering receptor expressed on myeloid cells 1 in patients with septic arthritis or rheumatoid arthritis. Ann Rheum Dis 2009, 68:1768-1774.

7. Yasuda T, Takeyama Y, Ueda T, Shinzeki M, Sawa H, Takahiro N, Kamei K, Ku Y, Kuroda Y, Ohyanagi H: Increased levels of soluble triggering receptor expressed on myeloid cells-1 in patients with acute pancreatitis. Crit Care Med 2008, 36:2048-2053.

8. Ramirez P, Kot P, Marti V, Gomez MD, Martinez R, Saiz V, Catala F, Bonastre J, Menendez R: Diagnostic implications of soluble triggering receptor expressed on myeloid cells- 1 in patients with acute respiratory distress syndrome and abdominal diseases: a preliminary observational study. Crit Care 2011, 15:R50.

9. Determann RM, Weisfelt M, de Gans J, van der Ende A, Schultz MJ, van de Beek D: Soluble triggering receptor expressed on myeloid cells 1 : a biomarker for bacterial meningitis. Intensive Care Med 2006, 32:1243-1247.

10. Determann RM, Schultz MJ, Geerlings SE: Soluble triggering receptor expressed on myeloid cells-1 is not a sufficient biological marker for infection of the urinary tract. J Infect 2007, 54:e249-250.

11. Bagshaw SM, Bennett M, Haase M, Haase-Fielitz A, Egi M, Morimatsu H, D'amico G, Goldsmith D, Devarajan P, Bellomo R: Plasma and urine neutrophil gelatinase-associated lipocalin in septic versus non-septic acute kidney injury in critical illness. Intensive Care Med 2010, 36:452-461.

\section{doi:10.1186/cc10533}

Cite this article as: Derive M, Gibot S: Urine sTREM-1 assessment in diagnosing sepsis and sepsis-related acute kidney injury. Critical Care 2011, 15:1?? 